\title{
LUMINESCENT PROBE FOR HIGHLY ENERGETIC COLLISIONS IN MIXTURES OF COMPLEX MOLECULES WITH ACCEPTORS OF VIBRATIONAL AND TRIPLET ENERGY*
}

\author{
G.A. Zalesskaya ${ }^{\dagger}$, D.L. Yakovlev, E.G. Sambor and D.I. Barangvsky \\ Institute of Molecular and Atomic Physics of the National Academy of Sciences \\ 70, Skariny Av., Minsk, Belarus, 220071
}

(Received July 23. 1998; revised version February 1, 1999)

\begin{abstract}
Pressure dependences of intensities and decay rates of time-resolved luminescence of acetophenone, benzophenone, anthraquinone were used to obtain the efficiencies of vibrational and triplet-triplet energy transfer. It was shown that vibrational relaxation of the chosen molecules can be interpreted in terms of two consecutive processes: rapid collisional relaxation of molecules from initially prepared states to a vibrational distribution at $T_{\text {vib }}$ by vibration-vibration process and relaxation of this vibrational distribution to the thermal one (vibration-translation process). At relatively small internal energy $<10000 \mathrm{~cm}^{-1}$, the collisional efficiencies of the vibration-vibration process in mixtures with polyatomic bath gases had values typical of processes with a supercollision contribution. Molecules relaxed from the upper vibrational level to the vibrational distribution after several collisions (2-3). The average energies transferred per collision are well correlated with predictions of the simple ergodic theory of collisional energy transfer. The majority of the collisions took part only in vibration-translation energy transfer of relatively small energies. The efficiencies of triplet-triplet energy transfer were analyzed for acetophenone, benzophenone and anthraquinone as donors and biacetyl-acceptor in a gas phase when energy of about $20000 \mathrm{~cm}^{-1}$ was transferred. It permitted us to elucidate the common features of highly energetic collisions. It was shown that the efficiencies are much lower than the gas kinetic ones and depended on the vibrational energy and temperature. It was discussed how to enhance triplet-triplet efficiencies due to vibrational excitation of a donor molecule.
\end{abstract}

PACS numbers: $31.50 .+\mathrm{w}, 34.50 . \mathrm{Ez}, 34.90 .+\mathrm{q}$

\footnotetext{
*The results of this paper were initially presented at The Jabloniski Centennial Conference on Luminescence and Photophysics, July 23-27, 1998. Torun', Poland.

${ }^{\dagger}$ phone: (375)(17)2841651; fax: (375)(17)2840030; e-mail: lsfm@imaph.bas-net.by
} 


\section{Introduction}

Collisional transfer of electronic and vibrational energy plays a key role in a great number of chemical and photochemical processes. Electronically and vibrationally excited molecules are intermediates in combustion, atmospheric chemistry, laser-induced reactions, surface etching. In real gas phase systems, the energy evolution depends on collisional energy transfer (CET). Intermolecular energy transfer is the important process competing with different chemical and photochemical processes, but in such complicated systems as vapors of polyatomic molecules, the nature of collisional effects has not been clearly understood up to now. Much experimental and theoretical attention has been focused on the nature of highly efficient collisions, the so-called supercollisions, which transfer large amounts of energy and occur in the case of large polyatomic molecules [1]. Many aspects of the problem investigated here have been discussed in Refs. [2-4].

Advances in laser technology created an opportunity for studying CET but only a restricted number of methods can be used for observation of CET in vibrationally excited polyatomic molecules. Luminescent methods first used to control collisional deactivation of highly vibrationally excited molecules [5] remain the most informative and successful up to now. In the present work as well as in Refs. [6-9] laser-induced luminescence has been used as a probe providing a sufficient time-resolution for research of collisional energy transfer between excited polyatomic molecules and bath gases. Laser-activated time-resolved luminescence of acetophenone, benzophenone and anthraquinone was employed to study the paths of triplet energy degradation. Due to its better time-resolution, the luminescent method permitted us to see more details of relaxation processes in polyatomics, in particular in studies of the nature of vibrational relaxation (vibration-vibration $(\mathrm{V}-\mathrm{V})$ and vibration-translation $(\mathrm{V}-\mathrm{T})$ energy transfers) as well as the competition of intermolecular vibrational relaxation witlr triplet-triplet energy transfer. The vibrational energy transfer quantities obtained by pressure dependences of intensities and decay rates of luminescence induced by $\mathrm{N}_{2}$ laser excitation of the ground electronic state molecules or by $\mathrm{CO}_{2}$ laser excitation of the previously prepared triplet molecules were compared.

The principle of measurement in the first series of experiments is as follows. A photon excites molecules to the first excited singlet state $S_{1}$. An efficient intersystem crossing (ISC) $S_{1} \leadsto T_{1}$ leads to trapping most of the molecules in the nearby long-lived triplet state $T_{1}$ and at the same time emission appears. Inert bath gases do not influence electronic relaxation processes. Therefore, the pressure dependences of luminescence decay rates and intensities allow us to study the vibrational energy transfer.

In the second series of experiments, the method of direct multiphoton $\mathrm{CO}_{2}$ laser excitation of triplet molecules was used to activate the delayed fluorescence [10]. When the vibrational equilibrium was established in triplet state, triplet molecules were re-excited by $\mathrm{CO}_{2}$ laser radiation (delay time is $10-20 \mu \mathrm{sec}$ ). Since the average vibrational energy of triplet molecules is comparable with the singlet-triplet energy gap $E_{\mathrm{ST}}$, thermally activated delayed fluorescence of these compounds in the vapor phase was intense. In all cases, the $\mathrm{CO}_{2}$ laser radiation induced additional pulses of delayed fluorescence and decreased phosphorescence. 
Emission of benzophenone $\left(\mathrm{C}_{13} \mathrm{H}_{10} \mathrm{O}\right)$, acetophenone $\left(\mathrm{C}_{8} \mathrm{H}_{8} \mathrm{O}\right)$ and anthraquinone $\left(\mathrm{C}_{14} \mathrm{H}_{8} \mathrm{O}_{2}\right)$ in the gas phase consists of spectrally overlapping delayed fluorescence and hot phosphorescence with equal decay times. These molecules are characterized by very fast ISC rates $S_{1} \leadsto T_{1}\left(k_{\mathrm{ST}}\right)$ of about $10^{10} \mathrm{sec}^{-1}$ for benzophenone [11, 12], anthraquinone [13] and $10^{12} \mathrm{sec}^{-1}$ for acetophenone [14] and large quantum yields (about unity due to $k_{\mathrm{S}} \cong k_{\mathrm{TS}}<k_{\mathrm{ST}}$, where $k_{\mathrm{S}}$ is the decay rate from $\left.S_{1}\right)$. For a small energy gap such as in benzophenone $\left(E_{S_{1}}=26200 \mathrm{~cm}^{-1}\right.$, $\left.E_{\mathrm{T}_{1}}=24000 \mathrm{~cm}^{-1}\right)$, acetophenone $\left(E_{\mathrm{S}_{1}}=27500 \mathrm{~cm}^{-1}, E_{\mathrm{T}_{1}}=25750 \mathrm{~cm}^{-1}\right)$ and anthraquinone $\left(E_{\mathrm{S}_{1}}=23750 \mathrm{~cm}^{-1}, E_{\mathrm{T}_{1}}=21750 \mathrm{~cm}^{-1}\right)$, the intermediately dense manifold of mixed states cannot act as a dissipative quasicontinuum and this provides the reversibility of ISC. We use the bath gas pressure low enough, so that vibrational relaxation in singlet manifold is negligible. Excitation of molecules to the $S_{1}$ state with a laser light is a convenient means for preparing vibrationally excited molecules in mixed singlet-triplet states with a known amount of vibrational energy $E_{\mathrm{vib}}=h \nu-E_{\mathrm{T}}$, where $h \nu$ is the excitation energy, $E_{\mathrm{T}}$ is the energy of the triplet state 0 -level.

The aim of the present work is to study the efficiency of collisional transfer of electronic and vibrational energy for mixtures of triplet polyatomic molecules with acceptors of vibrational and triplet energy using time-resolved luminescence. To gain insight into the path of the triplet energy degradation in gas-phase systems, the competition of intermolecular vibrational relaxation with triplet-triplet energy transfer is analyzed. In order to deduce the collisional efficiencies of the processes under study, the pressure dependences of their rates were measured. The collisional efficiency $\beta$ was evaluated as $\beta=K_{\text {col }} / Z$, since in the pressure interval used the rate constant satisfied the Stern-Volmer relation. Here $Z$ is the gas kinetic bimolecular collision rate constant that can be obtained on the assumption of the Lemnard-Jones or Stockmayer collision frequency. The experimental $\beta$ can then be related to an average energy transferred per collision $\langle\Delta E\rangle$ as $\Delta E=\beta(E-\bar{E})=$ $\beta E_{\text {vil }}$. Here $\bar{E}$ is the average energy at a temperature of experiment.

\section{Experimental}

All experiments were performed on a. laser pulse fluorimeter with a time-resolution of $10^{-8}$ sec. Excitation was produced by a pulsed $\mathrm{N}_{2}$ laser with a repetition frequency of $10 \mathrm{~Hz}$. The photomultiplier was used as a detector. For a spectral range of $400-500 \mathrm{~nm}$, where spectral characteristics of emission of the molecules under study were measured, the interference filters with a $1.5 \mathrm{~nm}$ pass band and cutoff filters were used. Signals were recorded by a digital oscilloscope connected to IBM PC. Data acquisition and storage were controlled by the computer. A further signal improvement was obtained by mathematical data processing of about 500 signals.

The pulsed atmospheric-pressure $\mathrm{CO}_{2}$ laser was used in our experiments. A laser beam was focused by lenses from blanched $\mathrm{Ge}$, passed through the cell beam to the beam of visible light. The IR beam energy was monitored both in front of the cell and behind it. The maximum energy emission by a $\mathrm{CO}_{2}$ laser pulse of $944 \mathrm{~cm}^{-1}$ was about $5 \mathrm{~J}$ in $100 \mathrm{~ns}$. The average number of vibrational quanta per triplet molecule was evaluated by the method described in Ref. [10]. 
The substances under studies were stored in the side arm of a $120 \mathrm{~mm}$ long, $30 \mathrm{~mm}$ diameter cylindrical heated quartz cell that was evacuated to less than $10^{-5}$ torr and kept at a constant temperature. Fluorescence was observed at the right angle to the laser beam through an appropriate window. The vapor pressure of polyatomics was controlled by the temperature of the thermostated side reservoir. The bath gases were added directly to the cell and conserved for at least $30 \mathrm{~min}$, to allow a formation of a homogeneous mixture. The bath gases which are usually liquid at room temperature were stored in a bulb on the vacuum rack and purified by freezing. The pressure of the bath gases which was as a rule above 0.25 torr was measured using an oil manometer.

\section{Results}

Experimental data can be summarized in the following way. Time-resolved luminescence of the molecules under study exhibited a bi-exponential decay behavior.

1. Emission decay rates of fast (Figs. 1, 2) and long (Fig. 3) components of benzophenone, acetophenone and anthraquinone emission linearly increased with their vapor pressure and with bath gas pressure over the range of 0-8 torr and followed the Stern-Volmer behavior.

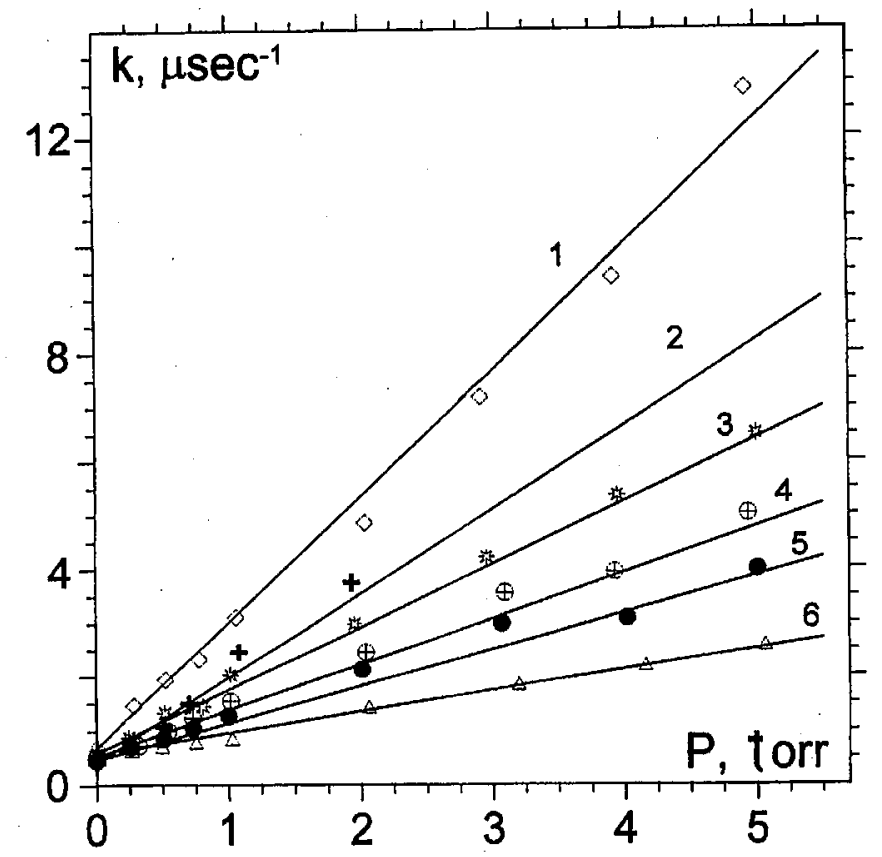

Fig. 1. Observed decay rates of fast components of anthraquinone emission as a function of bath gas pressure: $1-\mathrm{C}_{5} \mathrm{H}_{12}, 2-\mathrm{C}_{4} \mathrm{H}_{6} \mathrm{O}_{2}, 3-\mathrm{CCl}_{4}, 4-\mathrm{C}_{2} \mathrm{H}_{4}, 5-\mathrm{SF}_{6}$, $6-$ Ar. 


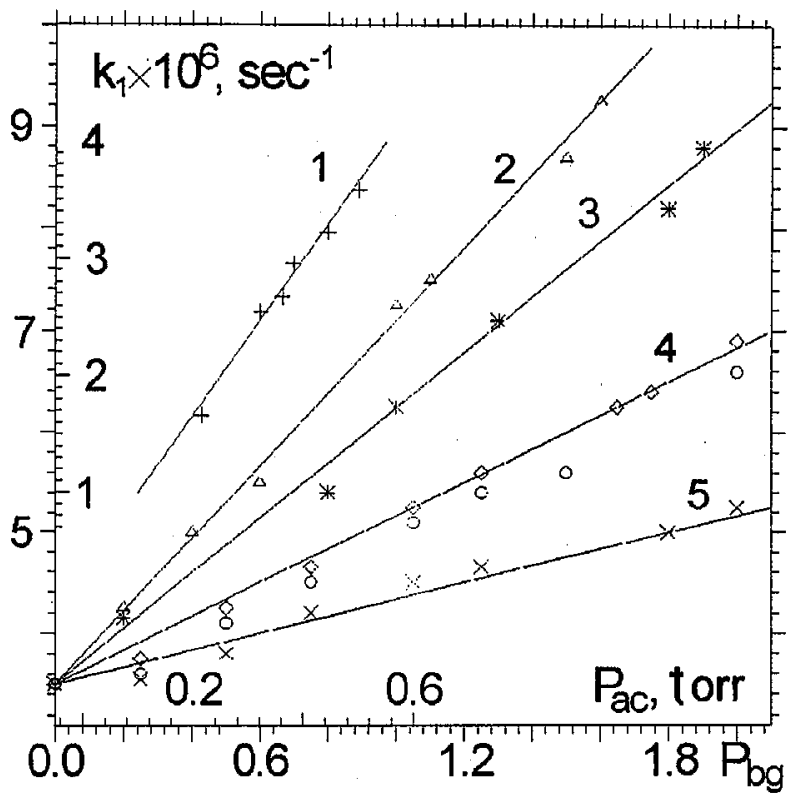

Fig. 2. Observed decay rates of fast components of acetophenone emission as a function of acetophenone (right scale of $k_{1}$ and upper scale of pressure) and bath gas pressure: $1-\mathrm{C}_{8} \mathrm{H}_{8} \mathrm{O}, 2-\mathrm{SF}_{6} ; 3-\mathrm{C}_{2} \mathrm{H}_{4}, 4-\mathrm{Ar}, 5-\mathrm{He}$.

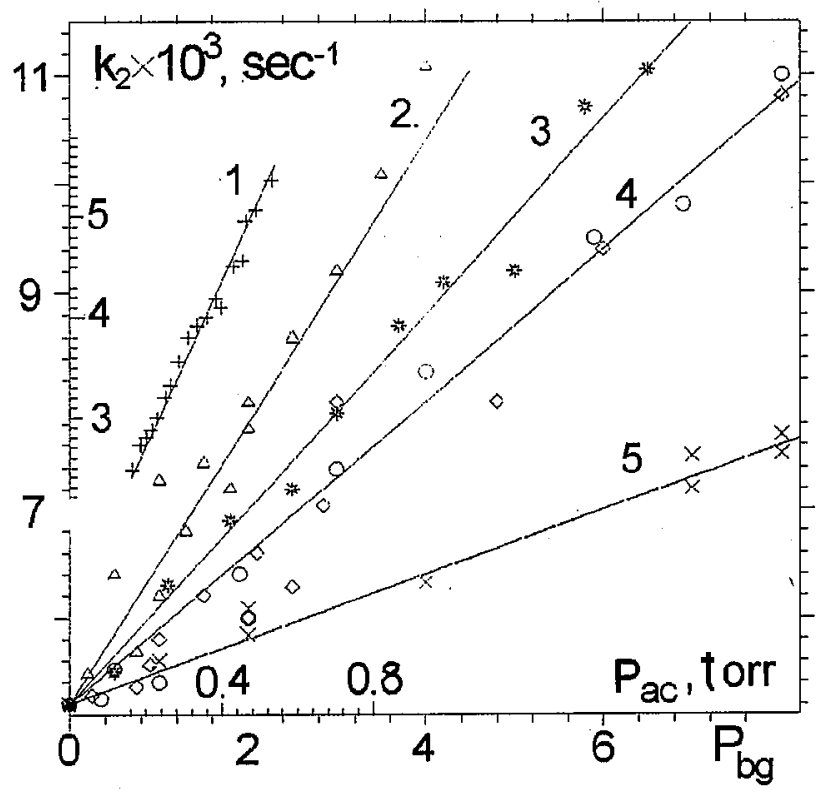

Fig. 3. Observed decay rates of slow components of acetophenone emission as a function of acetophenone (right scale of $k_{2}$ and upper scale of pressure) and bath gas pressure: $1-\mathrm{C}_{8} \mathrm{H}_{8} \mathrm{O}, 2-\mathrm{SF}_{6}, 3-\mathrm{C}_{2} \mathrm{H}_{4}, 4-\mathrm{Ar}, 5-\mathrm{He}$. 
2. The time-integrated intensities of the fast components decreased with bath gas pressure satisfying the Stern-Volmer relation

$$
I_{0} / I_{\mathrm{bg}}=1+P_{\mathrm{bg}} \beta Z \tau
$$

where $I_{0}$ and $I_{\mathrm{bg}}$ are the time-integrated intensities of luminescence for pure vapors and their mixtures with bath gases, respectively; $\tau$ is the collisionless lifetime of vibrationally excited molecules.

3. Over the range of 0-2 torr, time-integrated intensities of long components $\left(I_{2}\right)$ did not depend on the pressure of bath gases which cause only vibrational relaxation in triplet state. The integrated intensities ratio for a long time decay to that for the short one $\left(I_{2} / I_{1}\right)$ increased with bath gas pressure. This indicated a collisional mechanism which populates the long-lived states.

4. At a constant $\mathrm{CO}_{2}$ laser energy fluency, decay rates of $\mathrm{CO}_{2}$ laser-induced luminescence increased linearly with vapor pressure only in the limited pressure range (0.03-0.1 torr for acetophenone, 0.01-0.1 torr for benzophenone and anthraquinone). Collisional efficiencies for molecules under study did not exceed the values of $10^{-2}-10^{-3}$ even in the most favorable case of vibrational energy exchange between identical molecules. They decreased by one or two orders of magnitude for collisions with bath gases.

5. The dependences of anthraquinone luminescence decay rates on pressure of biacetyl (acceptor of the triplet energy) demonstrated some specific features as:

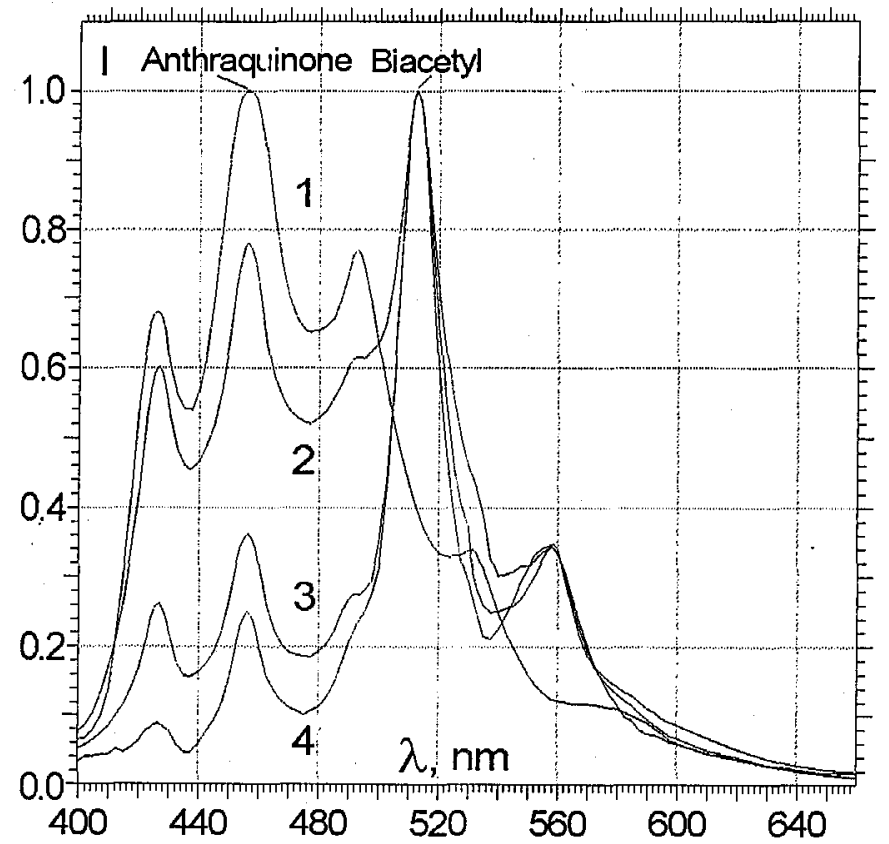

Fig. 4. Quenching of luminescence of anthraquinone and the increase in sensitized luminescence of biacetyl with biacetyl pressure at the selective $\lambda=337 \mathrm{~mm}$ excitation of anthraquinone. Biacetyl pressure: $1-0,2-0.13,3-0.68,4-2$ torr. 


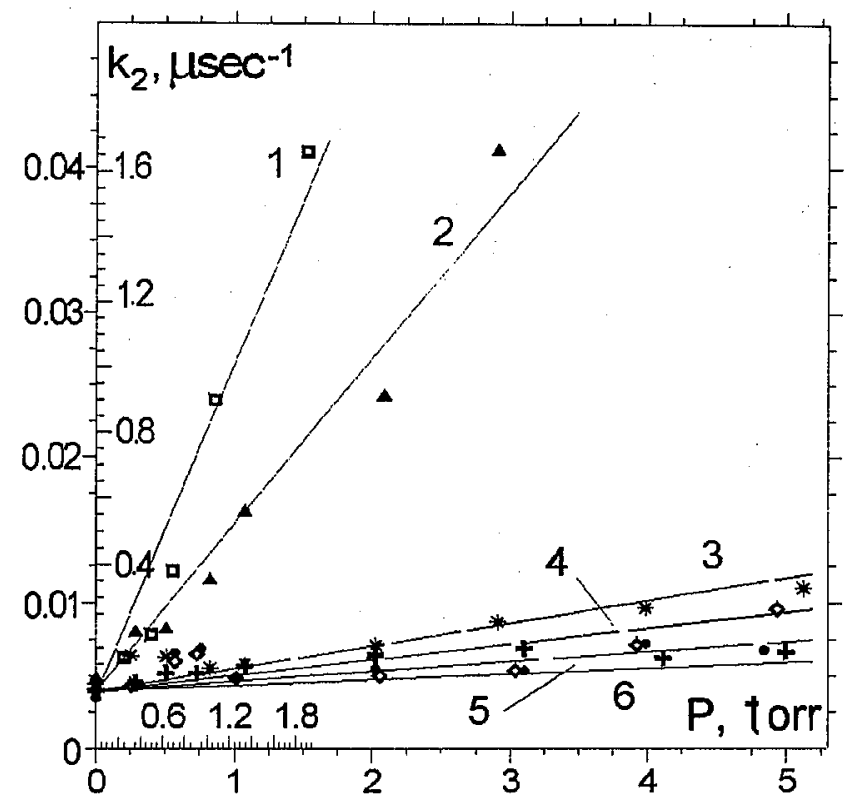

Fig. 5. Observed decay rates of slow components of anthraquinone emission as a function of bath gas pressure: $1-\mathrm{C}_{4} \mathrm{H}_{6} \mathrm{O}_{2}$ (right scale of $k_{2}$ and upper scale of pressure); $2-\mathrm{C}_{5} \mathrm{H}_{12}, 3-\mathrm{CCl}_{4}, 4-\mathrm{SF}_{6} ; 5-\mathrm{C}_{2} \mathrm{H}_{4} ; 6-\mathrm{Ar}$.

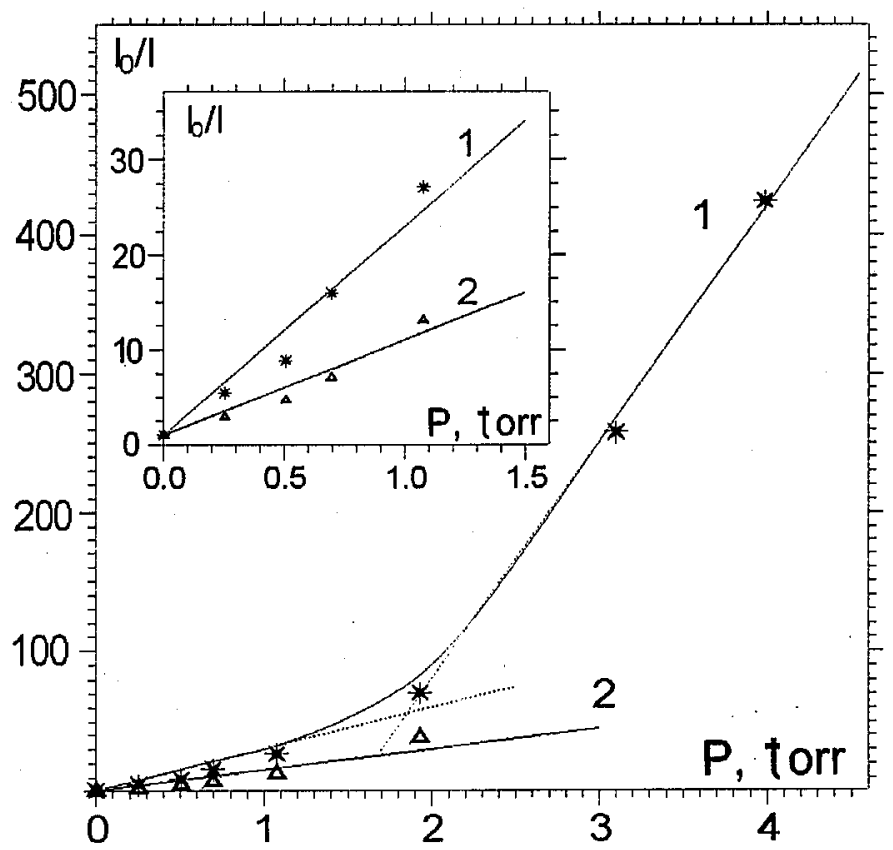

Fig. 6. Time-integrated relative intensity of donor-antraquinone $\lambda=455 \mathrm{~nm}$ emission versus biacetyl pressure: 1 - fast component; 2 - slow component. The insertion shows the initial stage of these dependences. 
(a) at a selective excitation of anthraquinone vapor at the $\lambda=337 \mathrm{~nm}$ radiation which was not absorbed by biacetyl, phosphorescence of biacetyl was observed. Intensity of this luminescence increased with biacetyl pressure (Fig: 4);

(b) while the decay rates of the fast components exhibited the dependence on the biacetyl pressure typical of vibrational energy transfer in mixtures with polyatomic bath gas (Fig. 1), the decay rates of the long components decreased with biacetyl pressure much faster than in the case of other bath gases (Fig. 5);

(c) phosphorescence quenching of anthraquinone by biacetyl in the coordinates of $I_{0} / I$ (the ratio of non-quenched to quenched time-integrated phosphorescence intensities at $\lambda_{\mathrm{ob}}=455 \mathrm{~mm}$ ) and $P$ (acceptor pressure) had different efficiencies in the pressure interval up to 1 torr and above it (Fig. 6).

\section{Discussion}

According to the photophysical properties of the investigated molecules, it is convenient to divide a triplet vibrational manifold into two energy regions. Below $E_{\mathrm{ST}}$, singlet--triplet separation energy, the triplet manifold includes the unmixed (pure) triplet vibronic states which are vibrationally relaxed. Above $E_{\mathrm{ST}}$ there are unrelaxed levels $B^{* *}$ and $B^{*}$ with a mixed singlet-triplet character $(|S\rangle-|T\rangle)$. Since in our case the vibronic levels are excited incoherently, the decay of luminescence can be interpreted by considering a simple kinetic description including a change in population due to, at least, four processes:

(1) $B^{* *}(|S\rangle-|T\rangle) \underset{k_{\mathrm{V}}^{-}}{\stackrel{k_{\mathrm{V}}}{\rightleftarrows}} B^{*}(|S\rangle-|T\rangle)$, rapid collisional relaxation of the vibrationally excited molecules with a rate $k_{\mathrm{V}}=k_{0}+k_{\text {col }} P=k_{\mathrm{V}} \exp \left(\Delta E / k T_{\text {vib }}\right)$ from the initially prepared mixed states to the distribution of excited vibrational states at $T_{\text {vib }}$.

(2) $B^{*}(|S\rangle-|T\rangle) \stackrel{k_{\mathrm{TS}}^{*}}{\longrightarrow} B|G\rangle$, irreversible ISC from the excited vibrational states to the dense ground state vibronic manifold.

(3) $B^{*}(|S\rangle-|T\rangle) \stackrel{k_{\mathrm{VT}}}{\longrightarrow} B|T\rangle$, relaxation of the vibrational distribution to the thermal one due to $\mathrm{V}-\mathrm{T}$ transfer with a rate $k_{\mathrm{VT}}=k_{0}^{\prime}+k_{\mathrm{col}}^{\prime} P$; back $\mathrm{V}-\mathrm{T}$ transitions can be ignored because of unfavorable values of $\Delta E / k T$, where $\Delta E=E_{\mathrm{ST}}>2000 \mathrm{~cm}^{-1}$.

(4) $B|T\rangle \stackrel{k_{T S_{0}}}{\longrightarrow} B|G\rangle$, simultaneously with the process (3) irreversible ISC from the vibrationally equilibrium states to the ground states vibronic manifold.

The system can be fitted by the well-known four-level kinetic scheme if we treat $B^{* *}(|S\rangle-|T\rangle)$ and $B^{*}(|S\rangle-|T\rangle)$ as single levels. Level 4 of this system represents the thermally equilibrated levels of the triplet manifold.

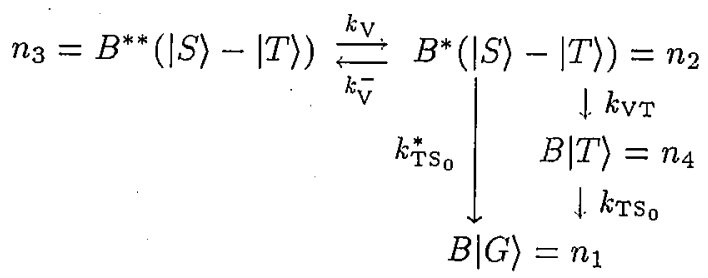


The necessary differential equations for the above scheme can be written as

$$
\begin{aligned}
& \frac{\mathrm{d} n_{3}}{\mathrm{~d} t}=-n_{3}+n_{2} k_{\mathrm{V}}, \quad \frac{\mathrm{d} n_{2}}{\mathrm{~d} t}=-n_{2}\left(k_{\mathrm{VT}}+k_{\mathrm{TS}_{0}}^{*}+k_{\mathrm{V}}\right)+n_{3} k_{\mathrm{V}}, \\
& \frac{\mathrm{d} n_{4}}{\mathrm{~d} t}=n_{2} k_{\mathrm{VT}}-n_{4} k_{\mathrm{TS}_{0}}, \quad \frac{\mathrm{d} n_{1}}{\mathrm{~d} t}=n_{2} k_{\mathrm{TS}}^{*}+n_{4} k_{\mathrm{TS}} .
\end{aligned}
$$

In the present case, the analysis of standard solution of these equations can be restricted to considering the changes in populations of levels $n_{3}$ and $n_{2}$ :

$$
\begin{aligned}
& n_{3}=A \exp \left(-k_{1} t\right)+B \exp \left(-k_{2} t\right), \\
& n_{2}=C \exp \left(-k_{1} t\right)+D \exp \left(-k_{2} t\right)
\end{aligned}
$$

with two decay components expressed. by Eq. (4):

$$
k_{1,2}=\frac{1}{2}\left[-\left(k_{\mathrm{V}}+k^{(2)}\right) \pm \sqrt{\left(k_{\mathrm{V}}-k^{(2)}\right)^{2}+4 k^{(3)}}\right]
$$

where $A=n_{3}^{(0)} \frac{-k_{2}-k_{\mathrm{V}}}{k_{1}-k_{2}}, B=n_{3}^{(0)} \frac{k_{1}+k_{\mathrm{v}}}{k_{1}-k_{2}}, C=n_{3}^{(0)} \frac{-k_{2}-k^{(2)}+k_{\mathrm{V}}}{k_{1}-k_{2}}, D=n_{3}^{(0)} \frac{k_{1}+k^{(2)}-k_{\mathrm{V}}}{k_{1}-k_{2}}$, $k^{(2)}=k_{\mathrm{VT}}+k_{\mathrm{TS}}^{*}+k_{\mathrm{V}}^{-}, k^{(3)}=k_{\mathrm{V}} k_{\mathrm{V}}^{-}$. Here $n_{3}^{(0)}$ denotes the initial population of level 3 produced by laser excitation at $t=0$ taken as a duration of a laser pulse (2.5 ns).

Our analysis was considerably simplified by noting that the vibrationally excited molecules relaxed to their equilibrium distribution via the $V-T$ process on a time scale much slower than the fast $V-V$ proress. As at our experimental conditions $\mathrm{V}-\mathrm{V}$ process passed with high efficiencies (nearly single collision conditions), the cascading of the molecules out of high vibrational levels to the lowest ones could be ignored. The energy gap between the initially populated level $n_{3}$ and the lowest level $n_{4}$ of the triplet state was greater than $5000 \mathrm{~cm}^{-1}$, therefore, relaxation from the higher levels by $\mathrm{V}-\mathrm{T}$ pathways was expected to be slow. Because of a large value of $k \mathrm{~V}\left(k_{\mathrm{V}} \gg k^{(2)}, k_{\mathrm{V}} \gg k^{(3)}\right)$, the second term of Eq. (4) can be approximated as $\left(k_{\mathrm{V}}-k^{(2)}\right)$ and then $k_{1}=-k_{\mathrm{V}}, k_{2}=-k^{(2)}=-\left(k_{\mathrm{VT}}+k_{\mathrm{TS}_{0}}^{*}+k_{\mathrm{V}}\right)$.

Thus, the increase in decay rates of the fast components with a pressure growth has been interpreted both in the earlier works [11-13] and in the present study as caused by an increase in vibrational $(\mathrm{V}-\mathrm{V})$ relaxation rates. As it has been shown above, these conclusions are supported by the pressure dependences of the integrated intensity of long $\left(I_{2}\right)$ and short $\left(I_{1}\right)$ components. The total intensities $\left(I_{1}+I_{2}\right)$ did not decreased in the bath gas pressure interval, in which $I_{1}$ decreased strongly, namely the collisional mechanism can populate the long-lived states.

The decay of slow components is due to V-T energy transfer and ISC from $T_{1}$ to $S_{0}$. In the case of luminescence excited by $\mathrm{N}_{2}$ laser, an addition of the bath gases at low pressures $\left(P_{\mathrm{bg}}<8\right.$ torr) results in increasing the decay rates of long components, that is, the $\mathrm{V}-\mathrm{T}$ process is rate determined. The pressure dependence of the decay rate of long components can be used to estimate the $\langle\Delta E\rangle$ values corresponding to $\mathrm{V}-\mathrm{T}$ CET. For example, the rate constants for the $\mathrm{V}-\mathrm{T}$. process obtained by Stern-Volmer plots of $K_{\mathrm{dec}}$ vs. $P_{\mathrm{bg}}$ have the following values: $2.4 \times 10^{-2} \mu \mathrm{sec}^{-1}$ torr $^{-1}, 8.3 \times 10^{-3} \mu \mathrm{sec}^{-1} \operatorname{torr}^{-1}$ and $\langle\Delta E\rangle-$ the values of 
$4.4 \mathrm{~cm}^{-1}$ and $1.8 \mathrm{~cm}^{-1}$ in pure benzophenone at $E_{\mathrm{vib}}=5670 \mathrm{~cm}^{-1}$ and acetophenone at $E_{\mathrm{vib}}=4123 \mathrm{~cm}^{-1} ; 2.25 \times 10^{-3} \mu \mathrm{sec}^{-1}$ torr $^{-1}$ and $\langle\Delta E\rangle=0.8 \mathrm{~cm}^{-1}$; $0.3 \times 10^{-3} \mu \mathrm{sec}^{-1}$ torr $^{-1}$ and $\langle\Delta E\rangle=0.2 \mathrm{~cm}^{-1}$ in mixtures of $\mathrm{Kr}$ with benzophenone and acetophenone, respectively, at the same $E_{\mathrm{vib}}$.

A comparison of these data with the results obtained at $\mathrm{CO}_{2}$ laser multiphoton excitation of triplet molecules has confirmed this interpretation. Essentially, the $\mathrm{CO}_{2}$ laser excitation method to measure the emission quantities has the lower time-resolution and thus it is possible to obtain only the V-T CET quantities as vibrational equilibrium establishes during the $\mathrm{CO}_{2}$ laser pulse $[7,8,10]$. The $\langle\Delta E\rangle$ values for $\mathrm{V}$-T CET obtained by two methods are found to be close. For instance, at $\mathrm{CO}_{2}$ laser excitation of benzophenone mixtures with $\mathrm{Kr}$ the $\langle\Delta E\rangle$ values vary from $2 \mathrm{~cm}^{-1}$ to $63 \mathrm{~cm}^{-1}$ with increasing $\mathrm{CO}_{2}$ laser energy density $E_{\mathrm{CO}_{2}}$ and, as consequence, $E_{\text {vib }}=\langle n\rangle h \nu_{\mathrm{CO}_{2}}$ from $3000 \mathrm{~cm}^{-1}$ to $12650 \mathrm{~cm}^{-1}$. Here $\langle n\rangle$ is the average number of $\mathrm{CO}_{2}$ laser photons absorbed per triplet molecule [10].

The experimental data demonstrate that after a few collisions with polyatomic gases vibrational equilibrium has been achieved. That is why, the version of the statistical model based on the ergodic assumption that after collision the excited and bath molecules are in microcanonical equilibrium, can be employed to estimate $\langle\Delta E\rangle$ for the $\mathrm{V}-\mathrm{V}$ CET process $[15,16]$ :

$$
\langle\Delta E\rangle=\frac{1}{\alpha_{1}+\alpha_{2}+2}\left[\left(\alpha_{1}+1\right)\left(E_{1}-b_{1}\right)-\left(\alpha_{1}+1\right)\left(\left\langle E_{2}\right\rangle_{\mathrm{T}}-b_{2}\right)\right],
$$

where the temperature dependences of vibrational heat capacities defining the values of $\alpha_{1}$ and $\alpha_{2}$ and average vibrational energy of the considered molecules defining the values of $E_{1}$ and $E_{2}$ are determined at a final temperature $T_{\mathrm{c}}$ estimated by the assumption of energy conservation after collisions. As it can be seen (Table I), a satisfactory correlation between the energy transfer parameters and the predictions by simple ergodic collision theory is found in the case of $\mathrm{V}-\mathrm{V}$ energy transfer.

The study of the triplet-triplet transfer permits one to gain insight into the paths of intermolecular energy transfer [17]. Moreo ver, it is a good example of the highly energetic collision process. For the pairs under study (benzophenone-biacetyl, anthraquinone-biacetyl), it is necessary to transfer energy of about $20000 \mathrm{~cm}^{-1}$ to activate acceptor biacetyl phosphorescence. Figure 4 shows the change of luminescence spectra with biacetyl pressure for the pair: anthraquinonedonor and biacetyl-acceptor. At a selective excitation of only anthraquinone vapors by $\lambda=337 \mathrm{~nm}$ radiation which is not absorbed by biacetyl, phosphorescence of biacetyl is observed with its intrinsic lifetime. The quenching rate has been monitored by recording the intensities of donor luminescence and phosphorescence of biacetyl as a function of the quencher pressure keeping the amount of anthraquinone at a constant value of 0.07 torr.

New details were found in the kinetic study of triplet-triplet transfer according to the data of Figs. 5 and 6 . An additional measurement of the triplet energy transfer from the vibrationally excited antraquinone-donor to biacetyl could be carried out. As seen from Fig. 6, over this range of biacetyle pressure the anthraquinone phosphorescence quenching by biacetyl in the coordinates of $I_{0} / I$ (the ratio of non-quenched to quenched time-integrated phosphorescence intensities) 
TABLE I

Data for collisions between excited molecules and bath gases.

\begin{tabular}{|c|c|c|c|c|c|c|}
\hline Collider & $\begin{array}{c}\Delta P \\
{[\text { torr] }}\end{array}$ & $\begin{array}{c}K_{\mathrm{col}} \\
{\left[\frac{1}{\mu \mathrm{sec} \cdot \text { torr }}\right]}\end{array}$ & $\begin{array}{c}Z \\
{\left[\frac{1}{\mu \mathrm{sec} \cdot \mathrm{lorr}}\right]}\end{array}$ & $\bar{\beta}$ & $\begin{array}{c}-\langle\Delta E\rangle_{\exp } \\
{\left[\mathrm{cm}^{-1}\right]} \\
\end{array}$ & $\begin{array}{c}-\langle\Delta E\rangle_{\text {theor }} \\
{\left[\mathrm{cm}^{-1}\right]}\end{array}$ \\
\hline \multicolumn{7}{|c|}{ Acetophenone } \\
\hline $\mathrm{C}_{8} \mathrm{H}_{8} \mathrm{O}$ & $0.17-0.53$ & 7.0 & 18.7 & $\begin{array}{l}0.37^{a} \\
0.28^{b}\end{array}$ & $\begin{array}{l}1525^{a} \\
1154^{b}\end{array}$ & $\begin{array}{l}1441^{c} \\
1439^{d}\end{array}$ \\
\hline $\mathrm{SF}_{6}$ & $0-1.5$ & 3.6 & 13.4 & $\begin{array}{l}0.268^{a} \\
0.291^{b} \\
\end{array}$ & $\begin{array}{l}1105^{a} \\
1200^{b} \\
\end{array}$ & $\begin{array}{c}518^{c} \\
1252^{d}\end{array}$ \\
\hline $\mathrm{C}_{2} \mathrm{H}_{4}$ & $0-2.0$ & 2.7 & 20.8 & 0.129 & 532 & $\begin{array}{c}285^{c} \\
1285^{d} \\
\end{array}$ \\
\hline $\mathrm{Ar}$ & $0-2.0$ & 1.6 & 14.9 & $\begin{array}{l}0.107^{a} \\
0.137^{b}\end{array}$ & $\begin{array}{l}441^{a} \\
565^{b} \\
\end{array}$ & $129^{c}$ \\
\hline \multicolumn{7}{|c|}{ Benzenophenone } \\
\hline $\mathrm{C}_{13} \mathrm{H}_{10} \mathrm{O}$ & $0.05-0.5$ & 9.6 & 26.2 & 0.366 & 2075.2 & $\begin{array}{l}2835^{c} \\
2086^{d}\end{array}$ \\
\hline $\mathrm{C}_{5} \mathrm{H}_{12}$ & $0-2.5$ & 2.98 & 22.8 & $\begin{array}{l}0.13^{a} \\
0.33^{b}\end{array}$ & $\begin{array}{c}741.1^{a} \\
1871.1^{b}\end{array}$ & $\begin{array}{l}2268^{c} \\
1047^{d}\end{array}$ \\
\hline $\mathrm{SF}_{B}$ & $0-8.0$ & 1.06 & 15.1 & $\begin{array}{l}0.070^{a} \\
0.210^{b}\end{array}$ & $\begin{array}{c}398.0^{a} \\
1190.7^{b}\end{array}$ & $\begin{array}{l}1113^{c} \\
1132^{d}\end{array}$ \\
\hline $\mathrm{Ar}$ & $0-8.0$ & 0.43 & 15.7 & $\begin{array}{l}0.027^{a} \\
0.054^{b}\end{array}$ & $\begin{array}{c}155.31^{a} \\
306.2^{b}\end{array}$ & $123^{c}$ \\
\hline \multicolumn{7}{|c|}{ Anthraquinone } \\
\hline $\mathrm{C}_{4} \mathrm{H}_{8} \mathrm{O}_{2}$ & $0.05-0.12$ & 7.0 & 47.0 & 0.148 & 1172 & $2930^{c}$ \\
\hline $\mathrm{C}_{4} \mathrm{H}_{6} \mathrm{O}_{2}$ & $0-1.2$ & 1.76 & 29.3 & $\begin{array}{l}0.06^{a} \\
0.18^{b}\end{array}$ & $\begin{array}{c}475 \\
1425\end{array}$ & $1242^{c}$ \\
\hline $\mathrm{C}_{5} \mathrm{H}_{12}$ & $0-5.0$ & 2.25 & 27.6 & 0.08 & 634 & $1176^{c}$ \\
\hline $\mathrm{C}_{2} \mathrm{H}_{4}$ & $0-5.0$ & 0.90 & 29.6 & 0.03 & 238 & $338^{c}$ \\
\hline $\mathrm{SF}_{6}$ & $0-5.0$ & 0.85 & 17.0 & 0.05 & 395 & $568^{c}$ \\
\hline $\mathrm{Ar}$ & $0-5.0$ & 0.40 & 19.6 & 0.02 & 158 & $172^{c}$ \\
\hline
\end{tabular}

${ }^{a}$ Experimental results are obtained from decay rate dependences on bath gas pressure.

${ }^{b}$ Experimental results are obtained from time-integrated intensity dependences on bath gas pressure.

${ }^{c}$ Approximation of Ref. [12].

${ }^{d}$ Approximation of Ref. [13]. 
TABLE II

Efficiencies of triplet-triplet energy transfer from vibrationally excited triplet molecules of biacetyl.

\begin{tabular}{c|c|c|c|c}
\hline \hline Donor & $E_{\text {mvib }}^{\text {don }}\left[\mathrm{cm}^{-1}\right]$ & Acceptor & $K_{\text {col }}\left[\mathrm{sec}^{-1}\right.$ torr $\left.^{-1}\right]$ & $\beta_{\mathrm{T}-\mathrm{T}}$ \\
\hline biacetyl & 2100 & anthracene & $1.88 \times 10^{7}$ & 0.65 \\
& 4250 & & $2.7 \times 10^{7}$ & 1.00 \\
\hline biacetyl & 2100 & naphthalene & $0.96 \times 10^{4}$ & $0.6 \times 10^{-3}$ \\
& 3680 & & $4.00 \times 10^{4}$ & $2.55 \times 10^{-3}$
\end{tabular}

and $P$ (acceptor pressure) had two approximately linear regions which were consistent with Eq. (1) where $\beta$ should be replaced by the efficiency of triplet-triplet energy transfer $\beta_{\mathrm{T}-\mathrm{T}}$. In the present case, the first region of this dependence corresponded to the pressure interval less than 1 torr where $\mathrm{V}-\mathrm{V}$ transfer took place, at higher pressures this process ceased. Both regions were analyzed to evaluate $\beta_{\mathrm{T}-\mathrm{T}}$. The calculations were performed using the collisionless lifetime values of $1.5 \mu \mathrm{sec}$ for the first stage and $200 \mu \mathrm{sec}$ for: the second one obtained from the pressure dependences of the decay rates. As the estimations had showed, the values of $\beta_{\mathrm{T}-\mathrm{T}}$ obtained from the dependences of $I_{0} / I$ on $P$ were 0.165 and 0.029 for the first and second stages, respectively, that is, triplet-triplet energy transfer was enhanced during $\mathrm{V}-\mathrm{V}$ relaxation in the triplet state of the donor.

In the second series of experiments, the preliminarily prepared triplet molecules of biacetyl were multiphoton-excited by $\mathrm{CO}_{2}$ laser radiation. For the pairs of biacetyl-donor and anthracene or naphthalene-acceptor the rates of triplet-triplet transfer were monitored by the variations in the decay rates of biacetyl emission as well as of anthracene and naphthalene ones as functions of $\mathrm{CO}_{2}$ laser energy density. The data obtained are summarized in Table II. Triplet-triplet energy transfers become more efficient as the vibrational energy of biacetyl increases.

Our data show that triplet-triplet energy transfer is enhanced during $\mathrm{V}-\mathrm{V}$ relaxation in the triplet state of the donor. We can compare them with the temperature effect on $\beta_{\mathrm{T}-\mathrm{T}}$ measured at equilibrium heating by Kotov and Pavlova [18]. The value of $\beta_{\mathrm{T}-\mathrm{T}}$ appeared to be temperature-dependent for exothermic triplettriplet transfer from biacetyl to anthracene. For temperature ranging from 363 to $463 \mathrm{~K}$ and average vibrational energy of biacetyl from 1350 to $2140 \mathrm{~cm}^{-1}$ the $\beta_{\mathrm{T}-\mathrm{T}}$ increased from 0.26 to 0.58 . In such a case the temperature dependence of $\beta_{\mathrm{T}-\mathrm{T}}$ was complex since the average vibrational energy of molecules and hot absorption of donor increased as well as the relative translational energy of the collision pair and, as consequence, the lifetime of donor-acceptor pair decreased. The mean lifetime of the excited donor-acceptor pair has been considered as one of the rate-determining factors in collisional energy transfer in the gas phase.

To elucidate the nature of the highly effective collisions it is very important to point out that the triplet-triplet energy transfer has been treated by applying the quantum mechanical description and it cannot be explained in the frame of classical approaches. Although the electronic interaction between the donor and acceptor 
governing the energy transfer process is weak $\left(\leq 50 \mathrm{~cm}^{-1}\right)$, as compared with the molecular electronic and vibrational energy, the collisions are accompanied by surprisingly large energy transfer, which takes place at a small interaction distance.

\section{Conclusions}

1. The intermolecular $\mathrm{V}-\mathrm{V}$ and $\mathrm{V}-\mathrm{T}$ relaxation processes for such large and complex molecules as acetophenone, benzophenone, anthraquinone are divided with the experimental time resolution of $10^{-8} \mathrm{sec}$. Two distinguished relaxation times are observed.

2. Vibrationally excited molecules relax to vibrational equilibrium during the fast stage of luminescence. The relaxation of molecules from upper levels occurs through fast $\mathrm{V}-\mathrm{V}$ exchange or transfer, which completes after several collisions in mixtures with polyatomic bath gases.

3. At relatively small internal energy $\left(E_{\text {int }}=9470 \mathrm{~cm}^{-1}\right.$ for benzophenone, $5760 \mathrm{~cm}^{-1}$ for acetophenone, and $11740 \mathrm{~cm}^{-1}$ for anthraquinone), the substantial amount of $\langle\Delta E\rangle$, typical of supercollisions, is transferred in collisions of vibrationally excited molecule with a cold one or with polyatomic bath. gases.

4. A correlation between the CET parameters and predictions by simple ergodic collision theory is found for the $\mathrm{V}-\mathrm{V}$ process.

5. Based on the $\mathrm{V}-\mathrm{T}$ data, it is concluded that the majority of energy transfer collisions involve $\mathrm{V}-\mathrm{T} / \mathrm{R}$ transfer of relatively small energies. A large difference in the values of $\langle\Delta E\rangle$ for $V-V$ and $V-T$ processes is obtained.

6. It is shown that at nonequilibrium donor excitation the efficiencies of triplettriplet transfer increase with a growth of vibrational energy of a donor.

\section{References}

[1] G.W. Flymn, C.P. Parmenter, A.W. Wodtke, J. Phys. Chem. 100, 12817 (1996).

[2] H. Hippler, J. Troe, in: Bimolecular Collisions, Eds. J.E. Bagott, M.N.R. Ashfold, The Royal Society of Chemistry, London 1989.

[3] R.J. Gordon, Comments At. Mol. Phys. 21, 123 (1988).

[4] J. Oref, D.C. Tardy, Chem. Rev. 90, 1407 (1990).

[5] N.A. Borisevich, B.S. Neporent, Opt. Spectrosc. USSR 1, 536 (1956).

[6] G.A. Zalesskaya, A.E. Gololobov, Spectrosc. Lett. 26, 1351 (1993).

[7] G.A. Zalesskaya, D.L. Yakovlev, D.I. Baranovsky, E.G. Sambor, Spectrosc. Lett. 29, 1173 (1996).

[8] G.A. Zalesskaya, D.L. Yakovlev, D.I. Baranovsky, E.G. Sambor, Spectrosc. Lett. 29, 1703 (1996).

[9] G.A. Zalesskaya, D.L. Yakovlev, D.I. Baranovsky, E.G. Sambor, J. Prikl. Spectrosc. 65, 340 (1998).

[10] N.A. Borisévich, G.A. Zalesskaya, Spectrosc. Lett. 19, 113 (1986).

[11] D. Zevenhujzen, R. van der Werf, Chem. Phys. 26, 279 (1977). 
[12] R.M. Hochstrasser, H. Lutz, G.W. Scott, Chem. Phys. 24, 162 (1974).

[13] Y. Hirata, E.C. Lim, Chem. Phys. Lett. 71, 164 (1980).

[14] N.A. Borisevich, V.V. Grusinsky, Dokl. Akad. Nauk SSSR 175, 852 (1967).

[15] S. Nordholm, B.C. Freiser, D.L. Jolly, Chem. Phys. 25, 433 (1977).

[16] L.E.B. Borjesson, S. Nordholm, L.L. Anderson, Chem. Phys. Lett. 186, 65 (1991).

[17] V.L. Ermolaev, E.N. Bodunov, E.B. Sveshnikova, T.A. Shachverdov, Radiationless Transfer of Electronic Energy, Leningrad 1977, p. 311.

[18] A.A. Kotov, V.T. Pavlova, Izv. Akad. Nauk SSSR, Ser. Fiz. 44, 794 (1980). 